\title{
Control mechanisms in problem solving
}

\author{
JOHN SWELLER \\ University of New South Wales, Kensington, New South Wales 2033, Australia
}

\begin{abstract}
Hypothesig-testing theories and information processing theories have both been used to explain the results of problem solving experiments. The theories have, in general, been applied to different classes of problems, resulting in little overlap or interaction between them. Information processing theories have tended to emphasize problems in which the problem solver's primary source of information concerning the correctness of his moves is the problem goal and, as a result, the goal plays a major role in the control mechanisms determining moves. Hypothesis theories have emphasized problems in which direct feedback is the primary control mechanism available. It is suggested that altering a problem solver's major source of information from a goal to feedback or vice versa will have important implications for the course of problem solving and what is learned during the solution process. Specifically, changes in the information feedback and/or goal-specificity characteristics of a problem should, in a predictable fashion, alter problem solving strategies, which should, in turn, through the presence or absence of information compression devices such as rule induction, strongly affect transfer. Experiments using the Tower of Hanoi and visual maze-tracing problems indicated that altering the density of subgoals resulted in alterations in subsequent transfer performance.
\end{abstract}

A wide variety of tasks studied in the problem solving laboratory are characterized by decision-making processes. Solution is attained by a series of decisions. Two major classes of theories have arisen to explain and predict experimental results: information processing theories and hypothesis theories. (It might be noted that although these labels are commonly used, they are not strictly descriptive, since both theories discuss information processing.) Both theories arose and developed largely in isolation from each other, and although both emphasize decision making during problem solving, there appears to be little overlap either in the theoretical statements made or in the types of problems studied.

\section{Two Problem Types}

Hypothesis theory has been applied primarily to the stimulus-response-feedback type of problems, of which concept learning tasks are the best known. Subjects are presented a stimulus, such as one or more colored geometric shapes, and must decide which one of two or more possible responses to make (e.g., pointing to one of the objects) before being informed of the correctness of the response. Most stimulus-response-feedback problems require the induction of a rule for solution, and this has influenced the theories used to explain the results of experiments employing these types of problems. The most influential theory has been hypothesis-testing theory, with Levine (1975) providing the most comprehensive statement. In essence, the theory assumes that subjects solve problems by testing a series of hypotheses

I wish to thank Marvin Levine for extended discussions and comments on an earlier version of this paper. Requests for reprints should be sent to John Sweller, School of Education, University of New South Wales, P.O. Box 1, Kensington, New South Wales 2033, Australia. (or rules), with feedback on each trial determining whether a hypothesis will be rejected or retained.

Problems in another category, labeled transformation problems by Greeno (1978), require an initial state, a goal state, and permissible operators to transform the initial state into the goal state. A number of problems conform to this paradigm. The Tower of Hanoi or pyramid puzzle (see Anzai \& Simon, 1979; Cook, 1937; Gagne \& Smith, 1962; Sweller, 1976), missionariescannibals-type problems (see Greeno, 1974; Reed, Ernst, \& Banerji, 1974; Thomas, 1974), and water-jar-type problems (see Atwood \& Polson, 1976; Luchins, 1942; Sweller \& Gee, 1978) have all been employed in problem solving research over many years.

Information processing theories have been used to describe the structure and processes of transformation problems. A major mechanism used to explain the problem solving process is means-ends analysis. Its fundamental axiom is the following: Operators are selected at each problem state that will reduce the differences between the problem state and the goal state (see Newell \& Simon, 1972).

There are two major differences in the problem solving tasks primarily addressed by the theories. These are concerned with, first, the goal characteristics of the two classes of problems and, second, the feedback information provided to subjects during problem solving.

The goal in transformation problems is itself normally a problem state and, as such, highly specific. Its precise properties and characteristics are normally communicated to the problem solver before the problem solving episodes commence (although this is not essential; see Sweller \& Levine, 1982). The goal of stimulus-responsefeedback problems, on the other hand, normally is communicated to subjects only in the most general form. It 
probably can best be specified as "find the appropriate rule that will result in consistent positive feedback."

With respect to feedback, transformation problems normally do not provide subjects any totally unequivocal information concerning the appropriateness of their moves. They consequently may not have a record, either in memory or in writing, of appropriate move sequences. Attaining the goal will, of course, indicate that the previous moves were successful in mapping a route to the goal, although it may not necessarily have been the best route. This is the only feedback information normally available to subjects. In contrast to transformation problems, stimulus-response-feedback problems are normally extremely rich in feedback information, and thus, the possibility of a complete or partial record of previously correct responses exists.

While there are other differences between the two types of problems (e.g., differences in their stimulus characteristics), the above distinctions are of importance to present considerations. They can be summarized as follows: Transformation problems tend to have a goal clearly specified as a problem state but only general feedback concerning the accuracy of moves, whereas stimulus-response-feedback problems tend to have a goal that is not presented as a specific problem state but feedback that is highly specific concerning the accuracy of moves. We are now in a position to discuss the consequences of the distinctions and, in the process, to contrast the two theories of interest.

\section{Control Mechanisms}

Hypothesis theory is essentially a theory of rule induction. Hypotheses invariably refer to rules, providing a complete solution to the problem. It is for this reason that the theory was developed and tested using problems whose goal was a weakly specified rule from which a precise rule had to be discovered. A problem of this type can be solved only by a rule-induction, hypothesis-testing procedure, rather than an attempt to reach a clearly specified goal. The rich feedback environment provides people with information concerning the validity of possible hypotheses. It acts as an essential guide in the choice of hypotheses and, as such, is a major control mechanism, heavily determining the problem solving paths followed. Consequently, hypothesis theory emphasizes feedback assumptions, thus reflecting the importance of feedback to the theory.

In contrast, information processing theory in recent years has not emphasized rule induction, although it has considered rule induction (e.g., see Greeno, 1978; Hunt, Marin, \& Stone, 1966; H. A. Simon \& Lea, 1974). It has been heavily concerned with how people attain a clearly specified problem goal during solution of transformation problems, and, as will be discussed later, this rarely occurs by a rule-induction process. A version of means-ends analysis is the normal mechanism, and the occurrence or otherwise of a rule is irrelevant to the process. The presence of a goal is essential to all forms of means-ends analysis, and the more clearly specified is the goal, the easier it is to reduce differences between that goal and any given problem state. When subjects are unsure of the precise characteristics of the goal, reducing such differences becomes difficult.

A clearly specified goal effectively acts as a substitute for the feedback provided by stimulus-response-feedback problems in guiding subjects to a solution and is also a major source of control during problem solving. Consequently, when dealing with transformation problems, information processing theories place the same emphasis on the goal as hypothesis theories place on feedback. Both goals and feedback have a similar function of controlling the problem solving paths followed.

In summary, as a consequence of these different control mechanisms, theories dealing with stimulusresponse-feedback types of problems emphasize hypothesis testing because the control mechanism available to subjects, feedback, is assumed by the theory to operate on hypotheses and these represent the "core" of the problem solving process. The poorly specified goal cannot play a major role in guiding subjects through the problem. Theories dealing with transformation problems emphasize means-ends analysis because, in this case, the highly specific goal is a primary control factor, with means-ends analysis providing the major mechanism through which it acts in determining which moves are made. Since these problems rarely have clear feedback associated with every move, feedback cannot be an important component in guiding people through the problem.

\section{Problem Solving Strategies}

While the two theories tend to refer to different categories of problems, there is one category to which either theory could be applied. These problems, which will be referred to as "hybrid" problems, are distinguished by the fact that they can be solved either by rule induction or by a goal-oriented procedure such as means-ends analysis. They are invariably tranformation problems in which a sequence of moves that can be used to attain the goal is described by a rule. There are several examples of such problems, with the Tower of Hanoi probably being the best known (see H. A. Simon, 1975). While this problem is normally solved by means-ends analysis, it could be solved by rule-induction procedure, although the latter rarely occurs (see Egan \& Greeno, 1974).

The class of hybrid problems is large, since it includes all transformation problems in which the moves can be described by a rule. Many transformation problems can be turned into hybrid problems by, for example, specifying a goal that can be reached by a series of moves that are rule related.

The strategy used by problem solvers may be heavily affected by the particular control mechanism predominating in a given problem. If a person is presented a problem that provides little direct feedback concerning the accuracy of each move but which also provides a 
clearly specified goal, resulting in the goal providing the major control mechanism, then during the problem solving episode, one might expect the problem solver to take considerable note of future moves needed to attain the goal but to virtually ignore the previous moves that have resulted in the current problem state. Means-ends strategies reflect this division of attention by emphasizing differences between the current problem state and the goal state.

At the other extreme, we have problems that provide complete feedback concerning the accuracy of each response but a goal that is specified only in the most general sense. Consequently, feedback rather than a goal provides the major control mechanism. A means-ends strategy in which subjects look forward toward the goal cannot be employed in any meaningful way in this type of problem, because a state representing the goal has not been specified. The problem solver must look backward at previous problem solving episodes and attempt to extract the appropriate rule, presumably by the mechanisms described by hypothesis theory. This will be called a history-cued problem solving strategy. We can consequently see that problems in which control is provided by strongly specified goals and weak feedback are likely to be solved by a means-ends problem solving strategy, whereas problems with weakly specified goals and strong feedback are likely to be solved by a historycued problem solving strategy, leading to rule induction.

While a means-ends strategy may be common in many hybrid problems due to their feedback and goal characteristics, these can be changed by altering the control mix, and this should alter subjects' strategy. The present paper concentrates on alterations in feedback. We may predict that increases in feedback information during solution of a hybrid problem will increase the control provided by feedback and, consequently, increase the probability of a history-cued strategy being used. This, in turn, should increase the probability of rule induction occurring.

There are several ways in which feedback information may be increased, with the most obvious being to simply inform subjects whether each move is "correct" or "incorrect." A theoretically more interesting method is to vary the frequency of occurrence of subgoals. A problem solver, on reaching a subgoal provided by the experimenter or by the problem structure, is, in effect, informed that his previous moves were correct, and hence, he has a record (in memory and/or in writing, depending on procedure) of appropriate moves. In this sense, subgoals provide feedback information. An increase in the number of subgoals provided for any given problem will effectively increase the amount of feedback provided and thus increase the number of appropriate moves. Essentially, control is increased by additional subgoals to the point at which it can be equated with the effect of feedback on responses. At this point, it should have the same psychological consequences as conventional feedback, since the information provided will be similar or identical to feedback.
In summary, problems with few or no subgoals can be expected to be solved by a means-ends problem solving strategy. The goal in this case dominates the problem and, by acting as the major control mechanism, can be expected to blind problem solvers to the rule that could be used to solve a hybrid problem. Alternatively, increases in the number of subgoals can be expected to effectively increase the likelihood of problem solvers' employing a history-cued strategy, since a single goal is no longer the dominant source of information. A historycued strategy may allow rule induction to occur, and the problem may be solved primarily by this means rather than by conventional means-ends analysis.

\section{Transfer}

Assuming that a problem's structure and manner of presentation affect a problem solver's strategy as suggested, we must next ask what the effects are of solving a problem by rule induction as opposed to means-ends analysis. Transfer to subsequent problems may be heavily influenced by the manner of solution of previous problems.

The major transfer phenomena described in the problem solving literature (e.g., Einstellung, learning to learn, the sequence effect) invariably appear to involve problems that can be solved only by rule induction or hybrid problems that happen to be solved by rule induction. As befits a theory of rule induction, hypothesis theory has analyzed these phenomena in some detail. As was the case when dealing with single problems, the theory again posited mechanisms that assumed people used a history-cued problem solving strategy. In this case, the problem solver referred to previous problems rather than previous episodes within a problem. The subjects attempted to apply the type of rule used to solve previous problems to the current problem. If it was applicable, positive transfer would result. Negative transfer occurred when the rule appeared to be applicable but, in fact, was not (for detailed treatments of hypothesis theory and transfer, see Fingerman \& Levine, 1974; Levine, 1975; Sweller, 1980a, 1980b; Sweller \& Gee, 1978).

While transfer can be obtained using problems other than ones in which a rule has been induced, the range and size of the effects seem to be vastly reduced. There appear to be no readily obtained and replicated examples of negative transfer and few studies involving positive transfer (for an example, see Anzai \& Simon, 1979) using problems other than rule-induction or hybrid problems. Not only can transfer be readily and reliably produced using rule-induction problems, but negative transfer in the form of Einstellung can be massive: Extremely simple problems can be rendered effectively insoluble by the presentation of an appropriate series of preliminary problems (see Fingerman \& Levine, 1974; Sweller, 1980b).

The reasons for enhanced transfer (either positive or negative) under rule-induction conditions lies in the properties of rules. Before transfer can occur, some- 
thing that has been learned during the solution of one problem must act as a medium for transfer to other problems. A rule provides an ideal medium for this purpose. On the other hand, if a problem has been solved without anything being learned, there is also nothing to transfer. Theoretically, a problem solved by means-ends analysis could result in no learning at all, resulting in no transfer. Unlike rule induction, there is nothing intrinsic to the mechanism that necessitates learning. Nevertheless, the fact that some positive transfer has been observed without rule induction indicates that people are unlikely to solve a problem and learn nothing.

The implications of this analysis for hybrid problems are as follows. If a hybrid problem is solved by rule induction, large positive or negative transfer effects should be observable, and hence, with appropriate experimental paradigms, the conventional transfer phenomena should be obtainable. If, alternatively, the problems are solved by conventional means-ends analysis, transfer should be reduced or even eliminated.

\section{Alternative Formulations}

There are alternative formulations that may be contrasted with the present suggestions. Greeno (1980) distinguished between problem solving based on meansends analysis and problem solving based on knowledge based on planning. He suggested that problems can be solved more efficiently if problem solvers are given appropriate knowledge rather than required to use means-ends analysis. This may be correct under most circumstances. Nevertheless, if the present theorizing is valid, it is only under a knowledge-based problem solving strategy (in which knowledge may consist of a rule) that Einstellung will occur. The use of pure means-ends analysis may eliminate negative transfer, which must enhance the usefulness of this technique.

It should also be noted that Greeno, Magone, and Chaiklin (1979) have suggested a theory to explain Einstellung that places a much heavier reliance on goals. According to this theory, problem solvers adopt plans that guide search for a solution. Plans are associated with goals, and subgoals and are tested to see whether a particular plan is potentially capable of attaining a goal. Goals and subgoals consequently determine the problem solving steps taken. An inappropriate plan will generate Einstellung.

The extent to which problem solving steps are controlled by a goal or subgoals rather than by previous moves during an attempt to solve a problem while influenced by Einstellung is primarily an empirical question. Some evidence for a history-cued approach is, in fact, provided by Greeno et al. (1979). Examining subject protocols, they found some subjects who appeared to be activating plans without reference to goals. The present theory suggests this commonly occurs when problem solvers are influenced by set.
The present formulation also contrasts with that provided by H. A. Simon and Lea (1974). They suggest that information processing theory can be used to explain rule induction in the following manner. First, a dual problem space is employed rather than the single space conventionally used with transformation problems. A separate problem space is required for rules and for the instances to which the rules are applied. Problem solving in this case can be characterized as a search for a rule rather than a search for a problem solution. While the rules are generated in the rule space, they are tested in the instance space, and feedback from the test can then influence the rule generator. Simon and Lea suggest that problem solving procedures such as means-ends analysis are applicable to rule-induction problems, as well as to conventional transformation problems. The goal is a rule that conforms with all of the instances, and means-ends analysis is used to modify rule sets until this goal is attained.

H. A. Simon and Lea's (1974) formulation emphasizes a functional equivalence of the goal in transformation problems and the rule in the rule-induction problems. The present approach differs in that it emphasizes a functional equivalence of the goal and feedback. Both have a control function, leading problem solvers through the problem. Nevertheless, since the control mechanisms differ, subjects' knowledge of the structure of particular problems may also differ as a consequence. This may lead to radically different problem solving modes, partly because while, as pointed out above, learning is an inevitable concomitant of rule induction (an induced rule is a learned rule), it is not essential to problem solving involving means-ends analysis. Differential knowledge structures acquired after a problem is solved by rule induction or conventional means-ends analysis may be essential to any theory that incorporates both problem solving techniques.

The present formulation may be summarized by a long chain (see Figure 1) that can be used to predict the effect of subgoals or some other form of feedback information on transfer and transfer phenomena. An increase in the number of subgoals should increase the amount of valid information and hence alter the control mix away from sole reliance on the goal. This will increase the likelihood of a history-cued problem solving strategy's being used, which, in turn, should enhance the probability of rule induction. Rule induction should increase the size of transfer effects and increase the probability of obtaining specific transfer phenomena. This should contrast with a situation with no subgoals. The lack of feedback information should increase the probability of a means-ends strategy and, consequently, reduce the probability of rule induction and transfer.

Experiments 1 and 2 test these predictions using, respectively, two quite different hybrid problems: the Tower of Hanoi and visual maze tracing. Unrelated problems are used in the hope of indicating that the 
[subgoal density] affects [amount of information] affects [amount of control by the goal] affects [type of strategy (historycued, or meansends analysis)] affects [probability of rule induction being employed] affects [size of transfer effects] affects [probability of specific transfer phenomena (Einstellung, sequence effect, learning to learn, etc.)].

Figure 1. Chain linking subgoal density to transfer phenomena on hybrid problems.

preceding analyses have a considerable degree of generality.

\section{EXPERIMENT 1}

The present theoretical constructs were tested in Experiment 1 using the Tower of Hanoi as a test problem. As previously indicated, it is a hybrid problem normally solved without rule induction. There is considerable information available concerning its characteristics. H. A. Simon (1975), in a theoretical analysis, indicated several alternative strategies that can be used to solve the problem. Most of these strategies rely on means-ends analysis as a major mechanism. The "move-pattern" strategies, on the other hand, require subjects to discover a pattern of moves to reach the goal and, as such, involve rule induction and, according to the present analysis, a history-cued strategy. Anzai and Simon (1979), analyzing the protocols of a single subject repeatedly solving the Tower of Hanoi, found a strategy change from a forward search for the appropriate move to a backward (from the goal rather than the present problem state) search for appropriate subgoals. A degree of positive transfer was obtained due to the subject's learning a subgoal structure appropriate to the problem and chunking moves leading to a subgoal. A move-pattern strategy was not used.

Providing subjects with appropriate subgoals and ready access to a record of previous moves should increase the probability of a move-pattern rule's being induced and alter the transfer characteristics of the problem. As stated previously, subgoals can be used to vary the feedback information provided for each move. Increased subgoal densities effectively increase the amount of information. The differential effects of goals and feedback should, according to the present theory, disappear if the subgoal density is increased to the point at which there is essential equivalence between goals and feedback in terms of the amount of information provided for each move. Since, in addition, subgoals can be more readily presented and manipulated than feedback in the form of "correct-incorrect" statements following certain moves, this mode of information control was employed.

The following rule can be used to solve the Tower of Hanoi. The squares used to construct the tower are numbered from 1 to 5 in order of size, with the smallest labeled "1." The three locations (A, B, and C) are placed at the points of an imaginary triangle. Each square can hence be moved in a clockwise or anticlockwise direction. Assume that five squares must be moved from $A$ to $C$ and that $A$ to $B$ to $C$ to $A$ represents the clockwise direction. A rule that will provide solution in the minimum number of moves requires the following sequence in every group of four moves: (1) 1a, (2) $2 \mathrm{c}$, (3) $1 \mathrm{a}$, and (4) any other square larger than 2 . Numerals refer to square labels; " $\mathrm{c}$ " is clockwise, and "a" is anticlockwise. There is only one move in each group of four moves that conforms with "any other square larger than 2." Solution of the same problem using four squares rather than five requires that the direction of each move be reversed. If the rule is not so altered, the pyramid is reconstructed on Location B instead of Location C.

Experiment 1 was designed to observe the effects of alterations in the number of subgoals on problem solving strategies, rule induction, and transfer.

\section{Method}

Subjects. The subjects were 60 psychology undergraduates attending the State University of New York at Stony Brook.

Materials and Procedure. All subjects were required to move the pyramid of consecutively numbered $(1=$ smallest $)$ cardboard squares from Location $A$ to Location $C$ on every problem. Locations $\mathrm{A}, \mathrm{B}$, and $\mathrm{C}$ were arranged so that each of $\mathrm{A}-\mathrm{B}, \mathrm{B}-\mathrm{C}$, and C-A represented a clockwise move. Subjects had to record each move made in terms of the square moved $(1,2$, or 3 , etc.) and in terms of its direction ("c" for clockwise and "a" for anticlockwise). Four columns on paper were provided for this record, resulting in a complete record consisting of rows containing four moves each. The record was completed row by row rather than column by column. After completing each problem, subjects were permitted to study this record for $1 \mathrm{~min}$ before commencing the next problem.

Five groups of 12 subjects each were used. Group Subgoal 2 was required to solve four problems. The first two problems were identical preliminary problems requiring five squares to be moved. Fif teen subgoals were provided, which represents a subgoal after every 2 moves if no errors are made and the problem is solved in the minimum of 31 moves. A subgoal consisted of a description of an appropriate configuration of squares on the three locations. All 15 subgoals in the order that they had to be reached were continuously available to subjects. Subjects were told that each subgoal had to be passed. The third problem was the first critical problem and was identical to the preliminary problems except that subgoals were not available. The fourth problem was the second critical problem and consisted of four squares only. No subgoals were provided. The rule that could be used to solve the five-square problems was no longer appropriate on the four-square problem.

Groups Subgoal 5 and Subgoal 10 were identical to Group Subgoal 2, except that they were provided six and three sub- 
goals, resulting in a subgoal after every 5 and 10 moves, respectively (assuming solution in the minimum number of moves). Groups Square 5 and Square 4 were control groups required to solve a single five-square or four-square problem, respectively, under conditions identical to those used for the critical problems of the preceding groups. As a consequence, they had no access to subgoals.

Total time and total moves to solution were used as depen. dent variables. Subjects were informed that these dependent variables were relevant.

\section{Results and Discussion}

Mean moves and seconds to solution for the two critical problems are contained in Table 1. The 05 level of significance was used for all tests. Since moves and seconds were highly correlated, moves only will be discussed, except for cases in which the pattern of significant difference varied using seconds.

It is predicted on the basis of the foregoing theory that increases in the number of subgoals will increase speed of solving a subsequent problem that can be solved using the same or a similar rule (Problem Square 5) and decrease speed of solving a subsequent problem that cannot be solved using the same or a similar rule (Problem Square 4). A significant difference was obtained between groups on Problem Square $5[F(3,44)=7.50]$. The effect of subgoals was revealed by a Duncan range test, which indicated a significant difference between Groups Subgoal 2 and Subgoal 10. Increased subgoals resulted in increased speed of solution on this transfer problem. In fact, all Group Subgoal 2 subjects solved Problem Square 5 in the minimum of 31 moves. Range tests also indicated a significant difference between the control group, Group Square 5, and the experimental groups except Group Subgoal 10. Using seconds to solution, the control group differed from all three experimental groups.

With respect to Problem Square 4, a significant difference was again obtained between the groups $[F(3,44)=$ 5.12 ]. In this case, a decreased number of subgoals decreased the relative speed of problem solving, with Group Subgoal 10 requiring significantly fewer moves to solve the problem than Group Subgoal 2, again using a range test. Only Group Subgoal 10 differed significantly from the control group, Group Square 4.

This pattern of results may be interpreted as follows. Close subgoals on the initial problems shifted control away from the goal by providing problem solvers a sequence of moves that they knew were either fully or largely correct in enabling them to solve the problem. They were consequently more likely to refer to these moves (to use a history-cued strategy) when continuing to solve a problem or to solve new problems that they perceived as being similar. When the previously learned sequences of moves were appropriate, solution was very rapid; when it was inappropriate, solution was delayed (Group Subgoal 2). In contrast, a few distantly spaced subgoals were unable to shift control away from the goal to the same extent. There was little feedback associated with each move, preventing a history-cued strategy from developing. A means-ends strategy continued to be used, and rule induction did not occur.

Given this analysis, we must ask why minimal evidence of negative transfer by subgoal groups with many subgoals compared to the control group was obtained on Problem Square 4. The most probable reason concerns the structure of the Tower of Hanoi. By following the rule appropriate to the five-square problem, the pyramid ends on Location $\mathrm{B}$ instead of $\mathrm{C}$ in 15 moves. It is hence possible to arrive at $\mathrm{C}$ in 30 moves, which is not much longer than the mean of 27 moves required by Group Square 4 to attain solution using means-ends analysis. Five subjects in Group Subgoal 2 solved Problem Square 4 in precisely this manner. The remaining subjects abandoned the rule either before (in most cases immediately before) the pyramid was reconstituted on B or, in a few cases, after this point. There was, hence, limited scope for negative transfer.

The speed with which Group Subgoal 10 solved Problem Square 4 also requires comment. This group exhibited substantial positive transfer on this problem in comparison to all other groups. The transfer medium was unlikely to have been the move-sequence rule, since the rule required transformation for the foursquare problem and, in any case, there is no evidence that Group Subgoal 10 subjects induced this rule. Only one subject solved the preceding five-square problem in the minimum number of moves. The most probable transfer medium may have incorporated the chunking strategies described by Anzai and Simon (1979). Subjects may have learned as a single chunk the sequences of steps necessary to move a pyramid of squares, blocking an essential step (or subgoal) such as moving a large square from $\mathrm{A}$ to $\mathrm{C}$. This learning applies generally to all Tower of Hanoi problems. Learning how to construct,

Table 1

Mean Moves and Mean Seconds to Solution on Problems Square 5 and Square 4 in Experiment 1

\begin{tabular}{|c|c|c|c|c|}
\hline \multirow[b]{3}{*}{ Groups } & \multicolumn{4}{|c|}{ Problems } \\
\hline & \multicolumn{2}{|c|}{ Square 5} & \multicolumn{2}{|c|}{ Square 4} \\
\hline & Mean Moves & Mean Seconds & Mean Moves & Mean Seconds \\
\hline Subgoal 2 & 31.00 & 175.42 & 32.58 & 286.08 \\
\hline Subgoal 5 & 43.58 & 319.25 & 30.58 & 199.67 \\
\hline Subgoal 10 & 65.08 & 471.25 & 19.67 & 88.58 \\
\hline Square 5 & 85.58 & 890.25 & & \\
\hline Square 4 & & & 27.33 & 274.17 \\
\hline
\end{tabular}


rather than being given, a subgoal structure is also general. This type of learning is unlikely (due to redundancy) among subjects who have induced the movesequence rule, since this rule provides a complete solution to the five-square problem. The alternative strategies do not have this advantage of providing a complete solution to the problem unless they are learned perfectly, which is difficult due to their complexity. They do, nevertheless, have the advantage of generality.

\section{EXPERIMENT 2}

The theoretical constructs discussed are intended to have a high degree of generality. This can best be indicated by applying them to a problem that differs considerably from the Tower of Hanoi. Experiment 2 used visual maze-tracing problems. Unlike the conventional Tower of Hanoi, they are not necessarily hybrid prob. lems, since normally there is no rule that can be used to solve them. Nevertheless, they can be readily transformed into hybrid problems by constructing the mazes in such a way that solution can be attained by following some sort of regularity at each choice point. In Experiment 2, this was accomplished by having two paths leading from each choice point. One path was a continuation of the path entering the choice point, and the other path was at right angles to the entering path. The rule that allowed solution was to turn at each choice point rather than continue straight ahead. The same correct path was used for all preliminary problems. When drawn, it was essentially symmetrical, resembling a three-step pyramid with a mirror image immediately above it. One other point needs to be noted when comparing the Tower of Hanoi with visual mazes. When constructing the problem, there are substantially more degrees of freedom available to the experimenter using mazes. A maze can take any shape, whereas the Tower of Hanoi is essentially fixed. It is consequently easier to structure the tasks in such a way that they conform with those structures predicted by the theory to readily yield, for example, Einstellung. The difficulty faced when using the Tower of Hanoi, of finding a problem soluble by a different method and either insoluble or far more difficult to solve using the old method, is considerably reduced using mazes. There are many alternative problem structures available to the experimenter, allowing a more complete test of the theory. Clear-cut transfer phenomena should consequently be obtained in Experiment 2 .

\section{Method}

Subjects. The subjects were 48 psychology undergraduates attending the State University of New York at Stony Brook.

Materials and Procedure. Subjects were required to trace a path on a maze from a starting point to a goal using a red pen to leave a visible record of their moves. At each choice point, they could either turn or continue straight ahead, and they were required to write a " $T$ " below the maze if they turned or an
"S" if they went straight ahead. After reaching the goal, they hence had a list of Ts and Ss that they had to separately total before being presented the next maze. On problems requiring a turn at each choice point, this list consisted entirely of Ts if no incorrect moves had been made.

There were four groups of 12 subjects each. Group Subgoal 1 subjects were required to solve six problems each in the order in which they are described below. Problem 1 consisted of a maze only, soluble without error by turning at each of the 10 choice points. Each correct path leading from a choice point had a number next to it. The number following the first choice point was 1 , the second was 2 , and so on. These numbers represented subgoals, and subjects were informed that each had to be passed consecutively in order to solve the problem. Problem 2, which followed, was identical to Problem 1 except there were no subgoals. Subjects were informed of this relation before they were given Problem 2. Problem 3 had subgoals after each of its 12 choice points, and Problem 4 was identical, but without subgoals. Subjects were again aware of the relation between the two problems. While the mazes used on the first two and second two problems differed, the correct paths were identical. Hence, the differences were caused by differing deadend paths. Problem $\mathrm{Cl}$ was the first critical problem. The correct path was identical to the previous correct paths except that it contained 21 choice points. There were no subgoals. Problem C2, the second critical problem, had a path that was identical to the correct path of the preceding problem except that it led to a dead end just before the goal. The correct path led almost directly to the goal and required decisions at only three choice points. There were no subgoals.

Groups Subgoal 2 and Subgoal 0 were required to solve exactly the same problems except that Group Subgoal 2 had a subgoal after every second choice point on Problems 1 and 3 and Group Subgoal 0 had no subgoals on any problem. Group C1C2 was a contol group given Problem $\mathrm{C} 1$ followed by $\mathrm{C} 2$ without preliminary problems.

Seconds to solution was used as a dependent variable. Subjects were informed that the experimenter was noting solution times for each problem.

\section{Results and Discussion}

Mean seconds to solution for the two critical problems are presented in Table 2. The four groups differed significantly in solving Problem Cl $[\mathrm{F}(3,44)=14.66]$. Duncan range tests indicated that Groups Subgoal 1 and $\mathrm{C} 1 \mathrm{C} 2$ were significantly different from all other groups, including each other. The groups also differed in Problem $\mathrm{C} 2[\mathrm{~F}(3,44)=5.55]$. Range tests indicated that Group Subgoal 1 differed from Groups Subgoal 0 and $\mathrm{C} 1 \mathrm{C} 2$.

Evidence of rule induction may be used to indicate the extent to which moves were controlled by the goal or by feedback provided by subgoals. Nine Group

Table 2

Mean Seconds to Solution on Problems $\mathrm{C} 1$ and $\mathrm{C} 2$ in Experiment 2

\begin{tabular}{lcr} 
& \multicolumn{2}{c}{ Problems } \\
\cline { 3 - 3 } Groups & $\mathrm{C} 1$ & $\mathrm{C} 2$ \\
\hline Subgoal 1 & 153.00 & 133.92 \\
Subgoal 2 & 373.25 & 84.67 \\
Subgoal 0 & 389.92 & 30.92 \\
C1C2 & 788.42 & 36.25 \\
\hline
\end{tabular}


Subgoal 0 subjects made errors at some choice points on Problem C1. Despite the implicity of the rule, the power of the goal as a control mechanism has ensured the use of a means-ends strategy and prevented rule induction from occurring. Only one subject provided evidence of rule induction on Problem C2 by following the rule-based path. Clearly, for Group Subgoal 0, moves were generated by the goal rather than by the rule. In contrast, one Group Subgoal 1 subject made errors on Problem C1. Control shifted from the goal to the rule, presumably due to feedback provided by subgoals during the preliminary problems. A history-cued strategy was employed. Seven subjects continued to use this strategy on Problem C2 following the rule until they reached a dead end. For these subjects, control shifted away from the goal.

The pattern of results for the experimental groups precisely duplicated that of the results obtained for the experimental groups using the Tower of Hanoi. Increases in the number of subgoals on the preliminary problems heavily increased the relative speed of solving a subsequent problem for which the solution was similar to that required on the initial problems, but they retarded solution speed when an alternative solution was required. There are nevertheless, differences in the total pattern of results between the two experiments. These are restricted to the second critical problem. Specifically, Einstellung was obtained using visual mazes (compare Group Subgoal 1 with Group C1C2). The flexibility available when structuring mazes allows a solution for the initial problems to be devised that, if attempted on the critical problem, resulted in a massive increase in solution time over that obtained if the appropriate path is followed immediately. This flexibility is not available using the Tower of Hanoi. The same theoretical principles nevertheless seem to apply.

The present results only indicated Einstellung when very close subgoals had been used. A comparison of Groups Subgoal 0 and $\mathrm{C} 1 \mathrm{C} 2$ yields no evidence of the effect. In contrast, Luchins (1942) obtained the effect with visual mazes containing no subgoals. The reason for this appears to lie in his choice of mazes with a very small number (as low as one) of choice points. Under these circumstances, one would expect the goal itself to have the same function as subgoals in the present experiment. Information concerning appropriate moves is readily provided by the goal at all points on the maze.

The data provide evidence for the causal chain of Figure 1, linking information feedback with transfer phenomena such as Einstellung. Increases in feedback provided by subgoals shifted control of the problem solver's moves away from the goal of the problem, resulting in history-cued rather than means-ends strategies' being employed. This, in turn, increased the probability, of rule induction's being brought into play, resulting in increases in transfer. Einstellung was thus obtained. In contrast, little or no feedback emphasized the goal as the primary center of control. The meansends strategy employed as a consequence reduced the probability of rule induction and reduced transfer effects.

\section{GENERAL DISCUSSION}

This paper began by pointing out that two theories of problem solving, hypothesis-testing theory and information processing theories, appear to have minimal overlap, with each theory having developed somewhat independently and tending to deal with different categories of problems, history-cued rule-induction problems and problems solved primarily by means-ends analysis. We may conclude that these two categories of problems may be better considered as a single category whose important properties vary along two continua. These continua are specificity of the goal and strength of feedback. While both can vary independently, they tend to be negatively correlated, since both have a similar function: providing problem solvers with a guidance mechanism. Problems with a general goal and strong feedback have been emphasized by hypothesistesting theory, whereas information processing theorists have more frequently studied problems with a specific goal and weak feedback.

We may further conclude that direct feedback is a particular technique of providing problem solvers with information independent of the problem elements themselves and that a variety of alternative techniques to provide this information are available. The use of subgoals on previous problems was explored in the present study. The use of independent information results in problem solving strategies different from those used when subjects rely on problem elements such as the goal as a primary source of information. Different strategies give rise to different phenomena. In particular, a means-ends strategy is not likely to lead to significant transfer effects.

There are reasons for assuming that the essential process studied in the present experiments involves a shift in control from a goal to a learned construct and that subgoal density and rule induction are merely particular examples of techniques that are capable of accomplishing this shift. Anything at all that is learned by any method may have the same effect. Under these circumstances, transfer phenomena such as Einstellung may be obtainable under conditions that differ substantially from those that normally apply. Rule induction may be unnecessary, and even initial problem solving activity may be redundant.

Preliminary experiments carried out have supported this view. Prior to presentation of the Tower of Hanoi, subjects memorized a series of letters and numbers that corresponded to the moves of a successful, although lengthy solution to the problem. Subsequent problem solutions used by problem solvers tended to correspond 
to the previously memorized sequence and were consequently retarded compared to a group not required to memorize the sequence. Similar results were obtained using a maze. In this case, subjects were required to memorize a complex pattern prior to being presented the problem. The maze contained a pattern similar to the memorized one, except a break occurred before the finish point and, hence, it could not be used to solve the problem. Solution was again retarded due to subjects attempting to use the previously memorized information to solve the problem.

The present conclusions may have implications for other recent formulations. Larkin, McDermott, Simon, and Simon (1980) and D. P. Simon and Simon (1978) have suggested that expert problem solvers in a given area tend to work "forward," that is, to generate moves from a given problem state rather than the goal, whereas novices used means-ends analysis. This accords with the present formulations that, in addition, provide mechanisms for the transformation of a novice into an expert. A history-cued strategy in which moves were generated by the problem state attained, rather than by the goal, was used only after subjects had acquired a learned construct. The nature of previous activity determined whether the construct was acquired. It must, nevertheless, be noted that, in the present experiments, the "experts" who had had previous training of some sort (experimental as opposed to control groups) showed negative as well as positive transfer. This leads to the apparently anomalous prediction that experts are more likely to demonstrate Einstellung than are novices. Given the forward-working "expert" strategy described by Simon and Simon (1978), this must be considered possible.

\section{REFERENCES}

Anzal, Y., \& Simon, H. A. The theory of learning by doing. Psychological Review, 1979, 86, 124-140.

Atwood, M. E., \& Polson, P. G. A process model for water jug problems. Cognitive Psychology, 1976, 8, 191-216.

Cook, T. W. Amount of material and difficulty of problem solving. Journal of Experimental Psychology, 1937, 20, 288296.

Eann, D. E., \& Greeno, J. G. Theory of rule induction: Knowledge acquired in concept learning, serial pattern learning and problem solving. In L. W. Oregg (Ed.), Knowledge and cognition. Potomac, Md: Erlbaum, 1974.

Fingerman, P., \& Levine, M. Non-learning: The completeness of the blindness. Journal of Experimental Psychology, 1974, 102, 720-721.
Gaane, R. M., \& Smith, E. C. A study of the effects of verbalization on problem solving. Journal of Experimental Psychology, 1962, 63, 12-18.

Greeno, J. G. Hobbits and orcs: Acquisition of a sequential concept. Cognitive Psychology, 1974, 6, 270-292.

Greeno, J. G. Natures of problem solving abilities. In W. $K$. Estes (Ed.), Handbook of learning and cognitive processes (Vol. 5). Hillsdale, N.J: Erlbaum, 1978.

Greeno, J. G. Trends in the theory of knowledge for problem solving. In D. T. Tuma \& F. Reif (Eds.), Problem solving and education: Issues in teaching and research. Hillsdale, N.J: Erlbaum, 1980.

Greeno, J. G., Magone, M. E., \& Chaiklin, S. Theory of constructions and set in problem solving. Memory \& Cognition, $1979,7,445-461$.

Hunt, E. B., Marin, J., \& Stone, P. I. Experiments in induction. New York: Academic Press, 1966.

Larkin, J., McDermott, J., Simon, D. P., \& Simon, H. A. Expert and novice performance in solving physics problems. Science, 1980, 208, 1335-1342.

Levine, M. A cognitive theory of learning: Research on hypothesis testing. Hillsdale, N.J: Erlbaum, 1975.

Luchins, A. A. Mechanisation in problem solving: The effects of Einstellung. Psychological Monographs, 1942, 54(Whole No. 248).

Newell, A., \& Simon, H. A. Human problem solving. Englewood Cliffs, N.J: Prentice-Hall, 1972.

ReEd, S. C., ERngt, C. W., \& Banerui, R. The role of analogy in transfer between similar problem states. Cognitive Psychology, 1974, 6, 436-450.

Simon, D. P., \& Simon, H. A. Individual differences in solving physics problems. In R. S. Siegler (Ed.), Children's thinking: What develops? Hillsdale, N.J: Erlbaum, 1978.

Simon, H. A. The functional equivalence of problem solving skills. Cognitive Psychology, 1975, 7, 268-288.

Simon, H. A., \& LEA, G. Problem solving and rule induction: A unified view. In L. W. Gregg (Ed.), Knowledge and cognition. Potomac, Md: Erlbaum, 1974.

Sweller, J. The effect of task complexity and sequence on rule learning and problem solving. British Journal of Psychology, $1976,67,553-558$.

Sweller, J. Hypothesis salience, task difficulty and sequential effects on problem solving. American Journal of Psychology, 1980, 93, 135-145. (a)

Sweller, J. Transfer effects in a problem solving context. Quarterly Journal of Experimental Psychology, 1980, 32, 233 239. (b)

Sweller, J., \& Gee, W. Einstellung, the sequence effect and hypothesis theory. Journal of Experimental Psychology: Human Learning and Memory, 1978, 4, 513-526.

Swellen, J., \& Levine, M. The effects of goal specificity on means-ends analysis and learning. Journal of Experimental Psychology: Learning, Memory, and Cognition, 1982, 8, 463-474.

Thомаs, J. C., JR. An analysis of behaviour in the hobbitsorcs problem. Cognitive Psychology, 1974, 6, 257-269.

(Received for publication July 23, 1982; accepted October 18, 1982.) 